\title{
Truss Modular Beams with Deformation Energy depending on Higher Displacement Gradients
}

\author{
JeAn-JaCQUes Alibert \\ PIERRE SEPPECHER \\ Laboratoire d'Analyse Non Linéaire Appliquée, Université de Toulon et du Var 83957 La Garde \\ Cedex France \\ Francesco Dell'Isola \\ Dipartimento di Ingegneria Strutturale e Geotecnica Università di Roma La Sapienza Via \\ Eudossiana 18, 00184 Roma Italia
}

(Received 29 February 2000; Final version 7 February 2002)

\begin{abstract}
Until now, no third gradient theory has been proposed to describe the homogenized energy associated with a microscopic structure. In this paper, we prove that this is possible using pantographic-type structures. Their deformation energies involve combinations of nodal displacements having the form of secondorder or third-order finite differences. We establish the $\Gamma$-convergence of these energies to second and third gradient functionals. Some mechanical examples are provided so as to illustrate the special features of these homogenized models.
\end{abstract}

Key Words: Second gradient theory, third gradient theory, homogenization, $\Gamma$-convergence, finite differences, modular truss beam, pantograph

\section{INTRODUCTION}

A formalized theory for constitutive equations in continuum mechanics was first developed by Noll [1] (in particular papers 8 and 35). In the framework of the aforementioned axiomatization it was proven by Eringen [2] and Gurtin [3] that - if Cauchy materials are considered - the second principle of thermodynamics does not allow for any dependence of stress tensor on the second gradient of placement so that - in order to formulate a purely mechanical model in which constitutive equations involve such a second gradient - the new concept of interstitial working has to be introduced [4]. Enlarging the scope of the considered models, it has been possible to include the second gradient of placement in the set of admissible independent variables for constitutive equations also by adding at the same time further kinematical descriptors (e.g. directors modelling the microstructure or temperature) for the state of material particles as done, for example, in [5], [6] and [7].

However, another equivalent formalization of continuum mechanics, based on the principle of virtual power and stemming from the d'Alembert concept of mechanics, is possible (for a modern description of such a point of view see, for instance, [8] and [9]). 
Following the classification formalized by Germain [10], the mechanical material behaviour of bodies can be characterized by the expression of internal (deformation) energy in terms of the displacement gradients. Cauchy three-dimensional (3D) materials coincide with first gradient materials; their deformation is described by, and their deformation energy depends on, the first gradient of displacement only.

The deformation energy of second gradient $3 D$ materials, instead, depends also on the second gradient of displacement. Let us call $\varepsilon(\mathbf{u})$ the symmetric part of the gradient $\nabla \mathbf{u}$ of the displacement $\mathbf{u}$ and $\omega(\mathbf{u}):=\nabla \mathbf{u}-\varepsilon(\mathbf{u})$ its skew part. We say that a second gradient $3 \mathrm{D}$ material is incomplete if its internal energy depends only on $\nabla \mathbf{u}$ and $\nabla \omega(\mathbf{u})$. These materials are also called ' $3 D$ materials with couple stresses' (cf [5] and [11]): microrotations in these bodies are modelled by introducing in the constitutive equations the aforementioned dependence on $\nabla \omega(\mathbf{u})$. Such a modelling assumption has been subsequently improved by introducing microstructural kinematical descriptors (for more details see, for example, [6] and [12]).

Incomplete second gradient materials have been studied for a long time. The precursor of incomplete second gradient models is the elastica introduced by Euler, Bernoulli and Navier at the beginning of the eighteenth century: it is a one-dimensional (1D) model in which: (i) the attitude of the beam sections is kinematically described by the gradient of the vertical displacement field; (ii) the contact couple depends on the second derivative of the vertical displacement; (iii) the deformation energy depends on the gradient of the attitude and therefore on the second gradient of displacement.

Another example of a 1D model in which higher-order derivatives of displacement must be introduced is given by the theory of Vlasov (see, for example, [13], [14] and [15]) describing the twist of thin-walled beams. In Vlasov's homogenized model the phenomenon at the micro-level to be accounted for is the warping of beam sections and the corresponding deformation energy is shown to depend on the first and second gradients of the twist angle.

The first (incomplete) second gradient 3D model is due to E. Cosserat and F. Cosserat (at the beginning of the nineteenth century): in [16] the deformation energy explicitly depends on $\nabla \omega(\mathbf{u})$. More recently, incomplete second gradient materials have been introduced to model granular solids; for more details and for further references, see [17]. Complete 3D models have been introduced for describing capillary phenomena [18], [19]. These have begun to be extensively used in the theory of damage and plasticity (see [20], [21], [22] and [23]) as they provide a more accurate description of transition zones (e.g. of shear bands [24]) and, from a mathematical point of view, they lead to regularized well-posed problems. The regularizing properties of second gradient models are also exploited in the description of the mechanical behaviour of elastic crystals (see, for instance, [25]).

Complete and incomplete second gradient materials have fundamentally different behaviours. While, in incomplete models, boundary conditions fix the displacement and the rotation $\omega(\mathbf{u})$ (or their dual quantities of force and moment), in complete models one also has to fix $\varepsilon(\mathbf{u})$ or its dual quantity called double force [10] to which not all mechanicians are accustomed. Indeed, the only widely used double contact action is Vlasov's bimoment (see, for example, [15] and [26]) needed for describing the external action at the extremities of thin-walled twisted tubes.

It is remarkable that the mathematically established relationships between $1 \mathrm{D}$ or twodimensional (2D) second gradient models and Cauchy materials have been investigated only when the dependence of deformation energy on the second gradient of displacement can 
be related, at the micro-level, to variations of attitude. Indeed, the limit analysis 3D-1D or $2 \mathrm{D}-1 \mathrm{D}$ of plates or beams leads only to such types of second gradient model. Is there any fundamental physical reason for this? In our opinion, this is probably due to the desire to remain in a standard framework. For more details about these rigorous results, we cite [27], [28], [29], [30], [31], [32] and [33]. In technical theories of beams, which supply the mechanical grounds for the aforementioned mathematical results, the macro-models are related to micro-models using several identification procedures; for an extensive historical discussion we refer to Benvenuto [34], who traces back to Maxwell and de Saint-Venant [35] the first of these analyses. That which seems to be more encompassing is based on identification in expended power; one postulates a macroscopic and a microscopic model, a kinematic correspondence between the two deformations and assumes that the power expended in corresponding motions coincides. In this way one obtains, in terms of micro properties of the beam, the coefficients of the macro constitutive equations, the form of which has been postulated at the beginning (see, in particular, for truss modular beams [36] and [37]). The very nature of this procedure shows how the properties of the macro model, in general, are not obtained as a result of the homogenization process but are, instead, assumed a priori.

Here we present a microscopic model which leads to the simplest macroscopic second gradient model: the 1D planar beam already studied by Casal [19]. The structure we consider, i.e. the pantographic structure, is simple and the reader may have already experimented with it when handling a corkscrew. We assume that the considered pantograph is made of a very large number $n$ of small modules and we study its limit behaviour when $n$ tends to infinity. In other words, we study the homogenized model for the pantograph. The computation of the equilibrium state is straightforward and we prove rigorously, using the technique of $\Gamma$ convergence, that the homogenized model is really a second gradient model (section 3).

Considering different equilibrium situations, we recall in section 4.1 the principal features of this model and we obtain an evident and self-explanatory interpretation for its special features, in particular for the notion of double force.

Even though the general properties of third gradient materials have been studied by Mindlin and Tiersten [5] and Dillon and Perzyna [38], to our knowledge no homogenized third gradient model has been recognized as necessary for describing the behaviour of a truss structure. To find such a structure is a problem closely related to the previous problem. Indeed, once one has obtained a complete second gradient body, it is relatively easy to construct a third gradient body. We do this and describe a structure, based on the pantograph, which we call the Warren-type pantographic structure. Its homogenized energy is rigorously proven to correspond to the bending of a third gradient beam. This beam has an unusual behaviour which we describe briefly in section 4.2.

As the theorems and proofs are quite similar in both cases, we have decided to group them in a single theorem (section 3). This states that, for any $k>0$, a quadratic functional of finite differences of order $k$ converges to a quadratic functional of the $k$ th derivative. This convergence is proven in the sense of $\Gamma$-convergence with respect to the weak* topology of measures. In this way, our result does not depend on the choice of any interpolation of displacements which have physical meaning at nodes only. We identify the external forces which are admissible for the considered structure; it is a class of distributions of order $k$ which contains, in particular, any distribution of order $k-1$. For instance, for a second gradient beam $(k=2)$, the derivative of a Dirac distribution is admissible. This corresponds to the notion of punctual double force [10]. 


\section{J.-J. ALIBERT ET AL.}

In the mathematical literature, the problem of rigorous proof of convergence from refined models to homogenized models is widely addressed. The results we present in this paper are close to those found in [28], [39, [40] and [41].

\section{TRUSS BEAMS WITH PANTOGRAPHIC SUBSTRUCTURES}

In this section, we develop the mechanical heuristic considerations leading us to formulate the mathematical problem to be solved in the subsequent section. We introduce a modular pantographic structure and a Warren-type pantographic structure. We describe these at a micro-level as a truss constituted by Euler beams and find the deformation energy for it in terms of displacements of a finite set of nodes. The obtained expression is strongly suggestive from a mechanical point of view. Indeed, it has induced us to conjecture the following. If the dimension of the structure module dimension tends to zero and the number of beams tends to infinity, a macro model can be introduced in which (i) the displacements of the nodes are characterized by a (suitably regular) field and (ii) the deformation energy depends on second or third derivatives of thus introduced displacement field.

This conjecture, although mechanically well grounded, needs a mathematical proof. For a discussion on the relationship between the discrete and the homogenized models we refer to [27] and [28].

\subsection{The pantograph}

Let us consider a plane modular structure the module of which is constructed as shown in Figure 1.

We consider inextensible but flexible beams and refer to these by their endpoints. We call a structure made by two such beams $\left(B_{i}, D_{i+1}\right)$ and $\left(D_{i}, B_{i+1}\right)$, connected by a pivot at their common centre $C_{i}$, the $i$ th module. We consider the simplest possible geometry assuming that $\left(D_{i}, B_{i}, B_{i+1}, D_{i+1}\right)$ is a square.

The periodic structure (shown in Figure 2) made by $n$ such modules, the size of which is $n^{-1}$, is called the pantographic structure. The $i$ th module is connected to the $i-1$ th module by two pivots at $B_{i}$ and $D_{i}$. We assume that external forces and external constraints are applied at nodes $C_{i}$ only. Thus we need to express the deformation energy of the structure in terms of the displacement of the nodes $C_{i}$. We study the behaviour of the structure in the framework of linear elasticity. The deformation energy of an inextensible but flexible beam depends only on its transverse displacement. This energy is proportional to the square of the curvature. The computation of the equilibrium energy of a beam of length $\ell$ and flexural stiffness $K$, subject to given transverse displacements $a, b$ at the endpoints and $c$ at the centre is standard [14]. It reads

$$
\min \left\{\int_{0}^{\ell} \frac{K}{2} z^{\prime \prime}(x) \mathrm{d} x ; z(0)=a, z\left(\frac{\ell}{2}\right)=b, z(\ell)=c\right\}=\frac{6 K}{\ell^{3}}(a-2 c+b)^{2} .
$$




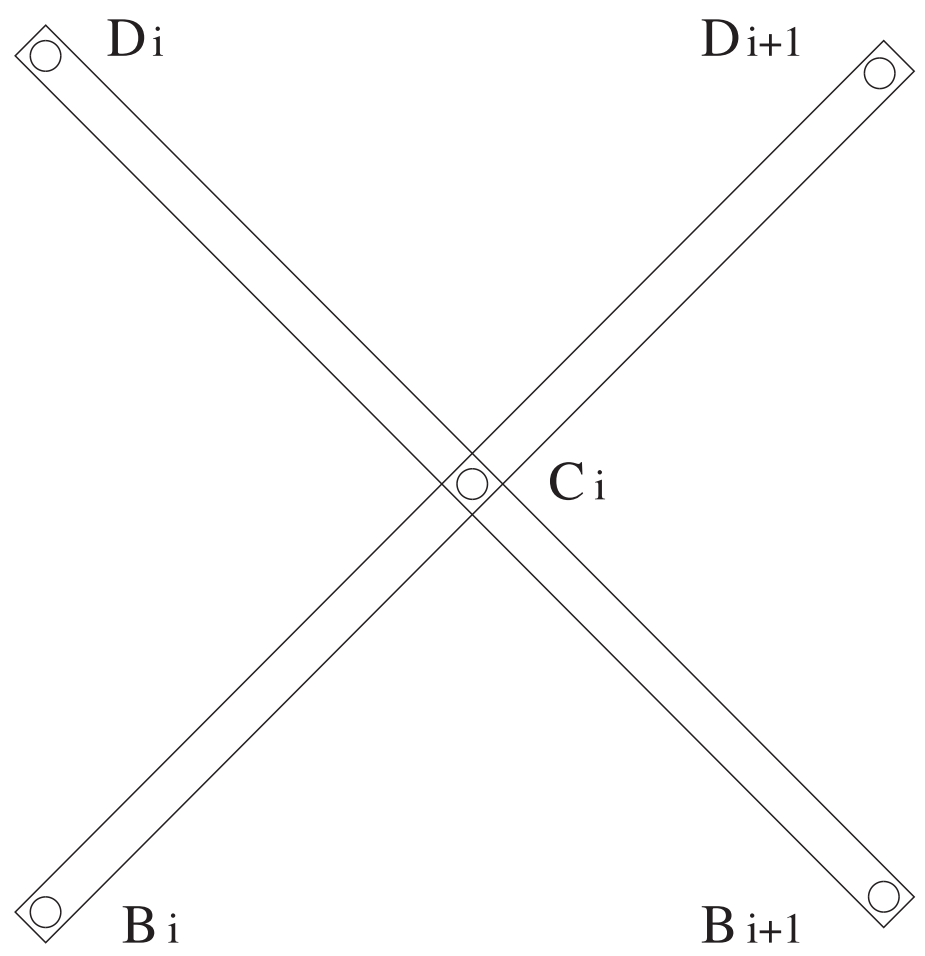

Figure 1. Module of the pantograph.

The length of all considered beams is $\ell=\sqrt{2} n^{-1}$. We assume that all beams $\left(B_{i}, D_{i+1}\right)$ (or $\left(D_{i}, B_{i+1}\right)$ ) have the same flexural stiffness denoted by $K_{f}^{+}$(or $K_{f}^{-}$).

Let us fix some notations adapted to the considered geometry. In the orthonormal basis $(x, y), x$ is the direction of the vector $C_{i} C_{i+1}$ (see Figure 2). We denote by $\mathbf{u}(M)$ the displacement of a generic node $M$ with respect to an unstressed reference configuration and by $(v(M), w(M))$ its components in the basis $(x, y)$. Then the transverse displacement for the beam $\left(B_{i}, D_{i+1}\right)$ is $(v-w) / \sqrt{2}$ while it is $(v+w) / \sqrt{2}$ for the beam $\left(D_{i}, B_{i+1}\right)$.

The equilibrium energy of each beam of the structure is then given by

$$
\begin{aligned}
& E\left(B_{i}, D_{i+1}\right)=K^{+} n^{3}\left[(v-w)\left(B_{i}\right)+(v-w)\left(D_{i+1}\right)-2(v-w)\left(C_{i}\right)\right]^{2} \\
& E\left(D_{i}, B_{i+1}\right)=K^{-} n^{3}\left[(v+w)\left(B_{i+1}\right)+(v+w)\left(D_{i}\right)-2(v+w)\left(C_{i}\right)\right]^{2}
\end{aligned}
$$

where $K^{ \pm}=(3 / 2 \sqrt{2}) K_{f}^{ \pm}$. As beams are not extensible, the displacement of nodes $B_{i}$ and $D_{i}$ is determined by the displacement of nodes $C_{i}$ and $C_{i-1}$. We have for all $i=2, \ldots, n$ :

$$
\begin{aligned}
2 v\left(B_{i}\right) & =(v+w)\left(C_{i}\right)+(v-w)\left(C_{i-1}\right) \\
2 w\left(B_{i}\right) & =(w+v)\left(C_{i}\right)+(w-v)\left(C_{i-1}\right)
\end{aligned}
$$




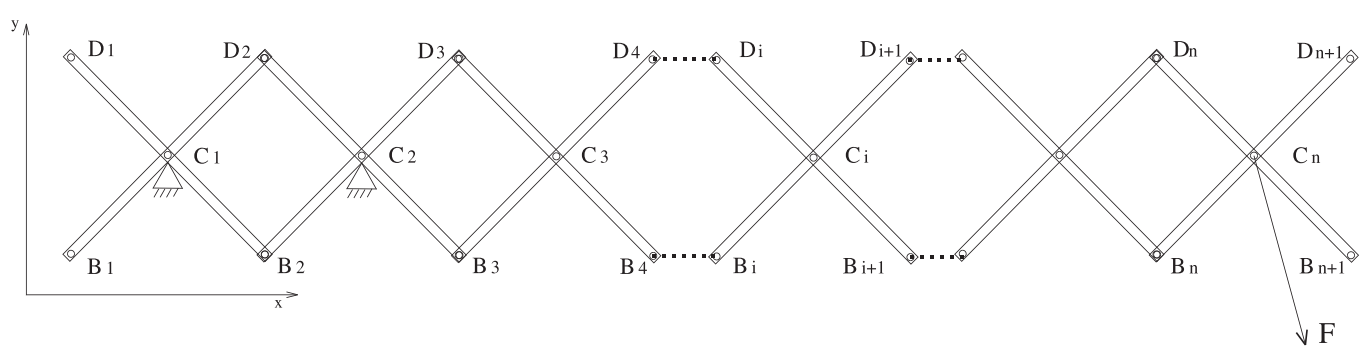

Figure 2. The pantographic structure.

$$
\begin{aligned}
2 v\left(D_{i}\right) & =(v-w)\left(C_{i}\right)+(v+w)\left(C_{i-1}\right) \\
2 w\left(D_{i}\right) & =(w-v)\left(C_{i}\right)+(w+v)\left(C_{i-1}\right) .
\end{aligned}
$$

Substituting Equations (2) into (1) we obtain for $i=2, \ldots, n-1$ :

$$
\begin{aligned}
& E\left(B_{i}, D_{i+1}\right)=\frac{K^{+}}{n}\left(n^{2}\left((v-w)\left(C_{i-1}\right)+(v-w)\left(C_{i+1}\right)-2(v-w)\left(C_{i}\right)\right)\right)^{2} \\
& E\left(D_{i}, B_{i+1}\right)=\frac{K^{-}}{n}\left(n^{2}\left((v+w)\left(C_{i-1}\right)+(v+w)\left(C_{i+1}\right)-2(v+w)\left(C_{i}\right)\right)\right)^{2} .
\end{aligned}
$$

We note that, due to our preceding assumptions, the endpoints $D_{1}, B_{1}, D_{n+1}$ and $B_{n+1}$ are free. We conclude that the first and the last two beams are undeformed. Their flexural energy vanishes so that the expression for the total deformation energy of the pantographic structure is

$$
E=\sum_{i=2}^{n-1}\left(E\left(B_{i}, D_{i+1}\right)+E\left(D_{i}, B_{i+1}\right)\right) .
$$

In the previous equations the reader can recognize the finite difference expressions for the second-order derivatives of $v-w$ and $v+w$. Therefore we can expect that, in the 1D beam model for a pantographic structure, the deformation energy is a quadratic functional of these derivatives. This statement is substantiated mathematically in section 3. Indeed we establish that the homogenized continuum model for the pantographic structure has the following deformation energy:

$$
\mathcal{E}=\int_{0}^{1}\left[K^{+}\left(\frac{\mathrm{d}^{2}}{\mathrm{~d} x^{2}}(v-w)\right)^{2}+K^{-}\left(\frac{\mathrm{d}^{2}}{\mathrm{~d} x^{2}}(v+w)\right)^{2}\right] \mathrm{d} x .
$$

In section 4.1 we discuss the mechanical implications of the model.

Let us determine the set of neutral displacements of the structure, i.e. displacements associated with the vanishing deformation energy. It is a four-dimensional vector space (in 


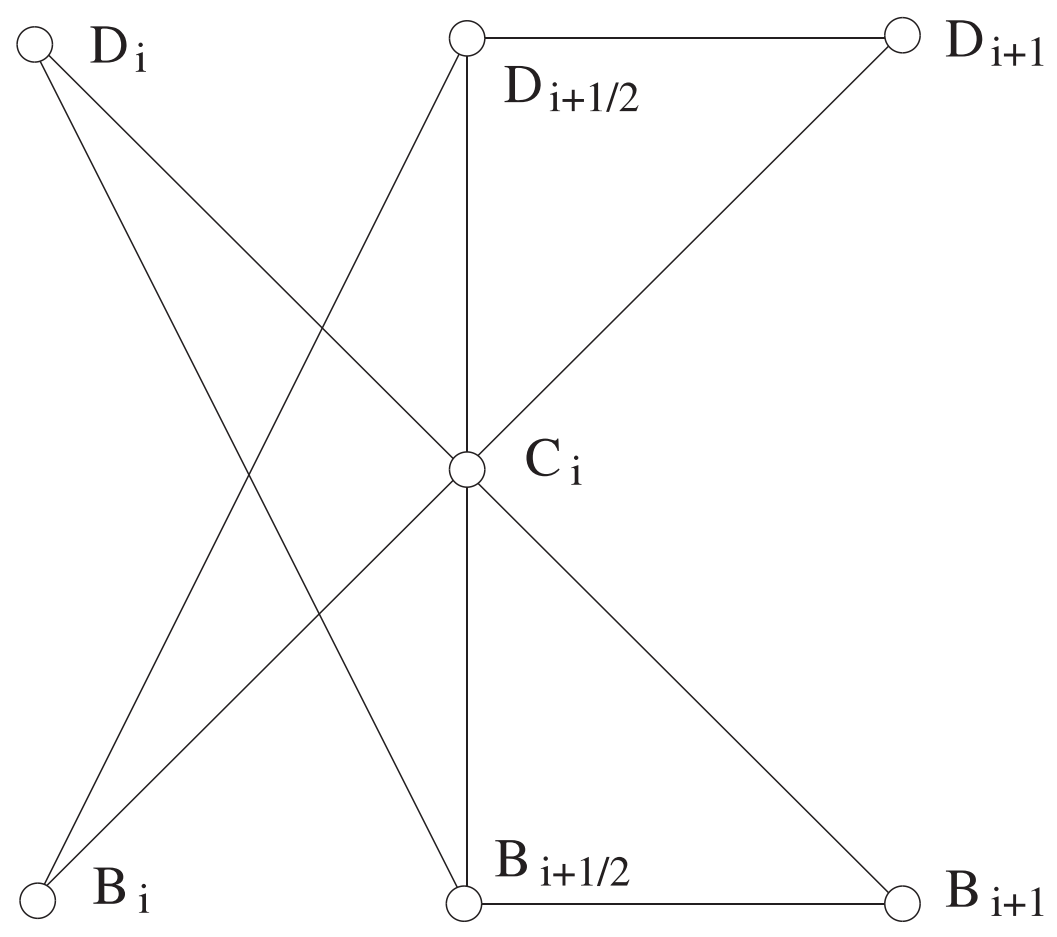

Figure 3. The pantographic truss structure.

such a circumstance one says that the structure has four degrees of freedom). Indeed, the structure is statically determined when the displacements of nodes $C_{1}$ and $C_{2}$ are prescribed. This can easily be checked by noting that no rigid displacement is allowed by applied constraints and that the number of constraint equations $(6 n)$ coincides with the degrees of freedom of constituting elements. The neutral set is generated by three rigid displacements and the uniform extension $\left(v\left(C_{i}\right)=i, w\left(C_{i}\right)=0\right)$.

We finally establish that a pantographic structure can be realized using a truss (i.e. a structure made only with extensible bars connected only through endpoints). Indeed each beam can be replaced by a shed truss; consider the modular truss, the module of which is presented in Figure 3 where all bars are inextensible except for bars $\left(D_{i+\frac{1}{2}}, D_{i+1}\right)$ and $\left(B_{i+\frac{1}{2}}, B_{i+1}\right)$. If their extensible rigidities are chosen to be $4 K^{+} n^{2}$ and $4 K^{-} n^{2}$ respectively, then the deformation energy of the considered truss is again given by Equation (4).

\subsection{Warren-type pantographic structure}

Let us now consider a Warren-type structure in which all upper bars are replaced by a unique pantographic structure. We study a plane modular structure, the module of which is shown in Figure 4.

This module is based on a pantographic module as described in the preceding section with the addition of four inextensible bars $\left(C_{i}, C_{i+\frac{1}{2}}\right),\left(C_{i+\frac{1}{2}}, A_{i}\right),\left(C_{i+\frac{1}{2}}, A_{i+1}\right)$ and $\left(A_{i}, A_{i+1}\right)$. 


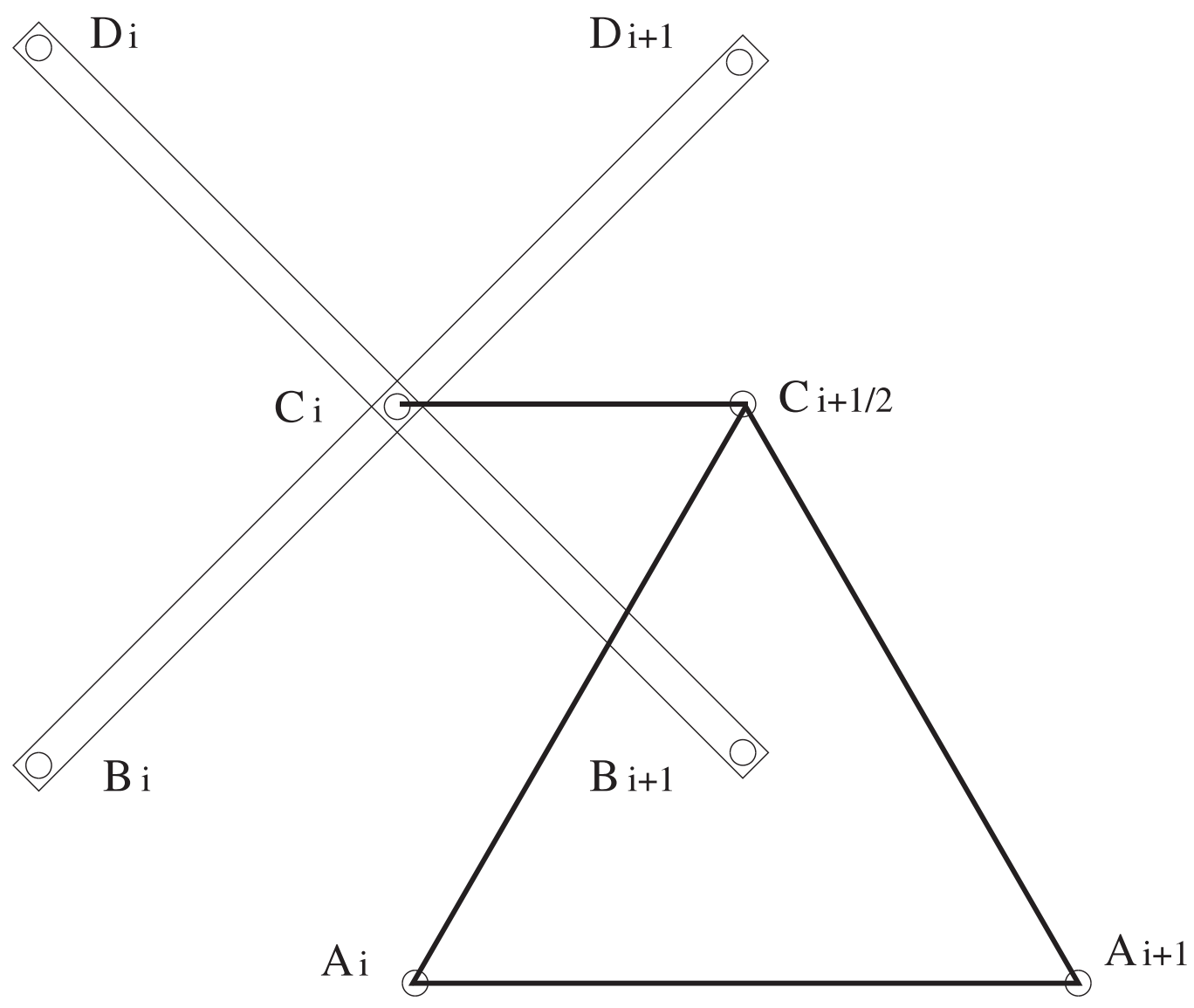

Figure 4. The Warren-type pantographic module.

For the sake of simplicity, we assume that all flexible beams have the same flexural rigidity, $K^{+}=K^{-}=K n^{2}$. We consider the simplest possible geometry assuming that $\overrightarrow{A_{i} C_{i}}=\overrightarrow{B_{i} D_{i}}$ and $\overrightarrow{B_{i+1} C_{i+\frac{1}{2}}}=\frac{1}{2} \overrightarrow{B_{i} D_{i}}$. The periodic structure made by $n-1$ such modules, the size of which is $n^{-1}$, is called the Warren-type pantographic structure. The $i$ th module is connected to the $i-1$ th module by pivots at $B_{i}, D_{i}$ and $A_{i}$. The first two modules are reinforced by two inextensible bars connecting the nodes $A_{1} C_{1}$ and $A_{2} C_{2}$ (see Figure 5).

We assume that external forces and external constraints are applied at nodes $A_{i}$ only. Thus we need to express the deformation energy of the structure in terms of the displacement of these nodes.

We use same notations as in the preceding section. Equations (2) are still valid. As the bars are not extensible, we have for all $i=1, \ldots, n+1$

$$
v\left(A_{i}\right)=v\left(A_{1}\right) .
$$

The displacement of node $C_{i+\frac{1}{2}}$ is determined by those of $A_{i}$ and $A_{i+1}$ : for all $i=1, \ldots, n$ 


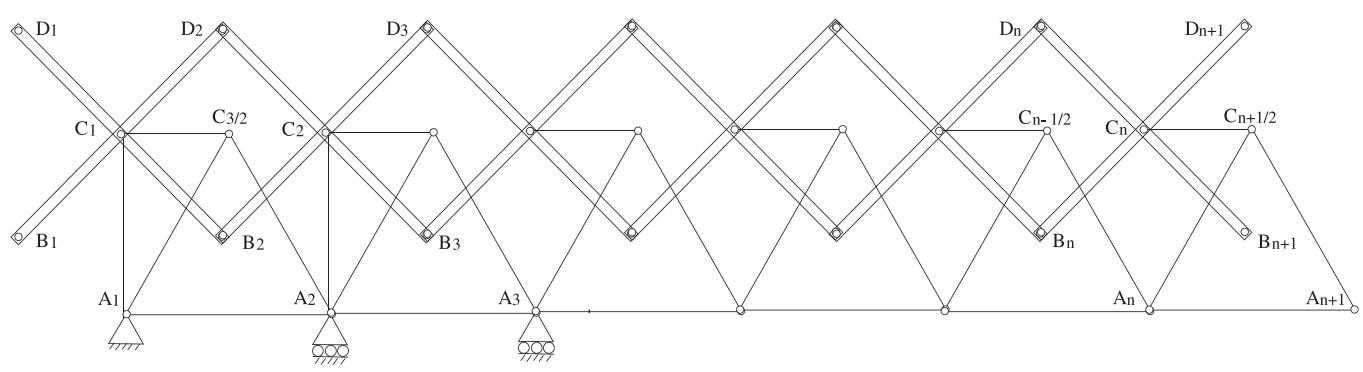

Figure 5. The Warren-type pantographic structure.

$$
\begin{aligned}
v\left(C_{i+\frac{1}{2}}\right) & =v\left(A_{1}\right)-w\left(A_{i+1}\right)+w\left(A_{i}\right), \\
w\left(C_{i+\frac{1}{2}}\right) & =\frac{1}{2}\left(w\left(A_{i+1}\right)+w\left(A_{i}\right)\right) .
\end{aligned}
$$

We also have, for all $i=1, \ldots, n$

$$
v\left(C_{i}\right)=v\left(C_{i+\frac{1}{2}}\right) .
$$

Moreover, in the first two modules, due to the added reinforcing bars

$$
w\left(C_{1}\right)=w\left(A_{1}\right), w\left(C_{2}\right)=w\left(A_{2}\right) .
$$

As the bars are not extensible, the deformation energy of the structure is concentrated in the substructure coinciding with a pantographic structure analogous to that studied in the previous section. This substructure is only subjected to constraints (9) and (10); the displacement of nodes $C_{1}, C_{2}$ and the displacement components $v\left(C_{i}\right)(i=3, \ldots n-1)$ are assigned. At equilibrium, $v\left(C_{i}\right)$ and $w\left(C_{i}\right)$ minimize the energy

$$
2 K n \sum_{i=2}^{n-2}\left[\left(v\left(C_{i-1}\right)-2 v\left(C_{i}\right)+v\left(C_{i+1}\right)\right)^{2}+\left(w\left(C_{i-1}\right)-2 w\left(C_{i}\right)+w\left(C_{i+1}\right)\right)^{2}\right]
$$

under the constraints (9) and (10). Thus we have

$$
w\left(C_{i}\right)=w\left(A_{1}\right)+(i-1)\left(w\left(A_{2}\right)-w\left(A_{1}\right)\right)
$$

and the equilibrium deformation energy reduces to

$$
F=2 K n \sum_{i=2}^{n-2}\left(n^{2}\left(v\left(C_{i-\frac{1}{2}}\right)-2 v\left(C_{i+\frac{1}{2}}\right)+v\left(C_{i+\frac{3}{2}}\right)\right)\right)^{2} .
$$

Using Equation (7) we obtain 


$$
F=2 K n^{-1} \sum_{i=2}^{n-2}\left(n^{3}\left(w\left(A_{i+2}\right)-3 w\left(A_{i+1}\right)+3 w\left(A_{i}\right)-w\left(A_{i-1}\right)\right)\right)^{2} .
$$

Here we can recognize the finite difference expression for the third-order derivatives of deflection and we expect that the 1D beam model for such a structure is described by a deformation energy which is a quadratic functional of this derivative. Indeed we establish, in section 3, that the homogenized continuum model for this structure has the following deformation energy:

$$
\mathcal{F}=\int_{0}^{1} 2 K\left(\frac{\mathrm{d}^{3} w}{\mathrm{~d} x^{3}}\right)^{2} \mathrm{~d} x
$$

In section 4.2 we discuss the mechanical implications of the model.

The set of neutral displacements of the structure is again a four-dimensional vector space. Indeed, the structure is statically determined when the displacement of node $A_{1}$ and the displacement components $w\left(A_{2}\right), w\left(A_{3}\right)$ are imposed. This can easily be checked by considering that no rigid displacement is allowed by applied constraints and that the number of constraint equations coincides with the degrees of freedom of constituting elements. The neutral set is generated by three rigid displacements and the uniform flexion $\left(w\left(A_{i}\right)=\right.$ $\left.i^{2}, v\left(A_{i}\right)=0\right)$.

The remark at the end of the previous section shows that the Warren-type pantographic structure can also be regarded as a truss modular beam.

\section{HOMOGENIZATION OF PANTOGRAPHIC STRUCTURES: MATHEMATICAL RESULTS}

In this section we prove that the sequence of discrete models (parameterized by $n$ in the previous section) for pantographic structures does converge to a continuous model generalizing Euler's elastica in the sense of De Giorgi's $\Gamma$-convergence (see [42], [43] and [44]). Thus, we establish a correspondence between the homogenized model and every element of the sequence of discrete models; when $n$ is suitably large the deformation energy in the limit model (corresponding to a fixed external action) is close to the corresponding energy for the discrete systems. The $\Gamma$-convergence, although involving rather abstract functional analysis concepts, supplies a convergence criterion having a clear mechanical basis.

\subsection{Mathematical formulation}

When a periodic structure is made of a large number $n$ of modules, it is natural to study the limit behaviour as $n$ tends to infinity. This is the homogenization procedure. We first need a functional framework in which we can define the displacement fields of the considered structures for all $n$, as well as their continuous limits. A displacement field for the pantograph or for the Warren-type structure (i.e. the displacement of each node $C_{i}$ or $A_{i}$ ) can clearly be identified with a vector valued function $(v, w)$ defined at points $i / n$ for $i=1 \ldots n$. In order 
to obtain a functional framework which does not depend on $n$ one could extend this function over the whole interval $[0,1]$ by considering, for instance, a piecewise linear extension or a piecewise constant extension [45]. Such extensions have no physical meaning and may influence the results. This is the reason why we prefer to associate to each component $v$ or $w$ (let us call it $u$ ) of the discrete displacement field, the discrete measure $u v_{n}$ where $v_{n}:=n^{-1} \sum_{i=1}^{n} \delta_{i / n}$ and $\delta_{i / n}$ denotes the Dirac mass concentrated at point $i / n$. Our problem is then naturally set in the space $\mathcal{M}$ of bounded Borel measures on the interval $[0,1]$.

For any positive integer $k$, let us denote by $A_{n}^{k}$ the set of those functions $u$ defined $v_{n}$ almost everywhere (i.e. at points $i / n$, for $i=1 \ldots n$ ) which satisfy

$$
u\left(\frac{i}{n}\right)=0, \quad i=1, \ldots, k
$$

We also denote by $H_{\text {left }}^{k}$ the set of those functions in the usual Sobolev space $H^{k}(0,1)$ which satisfies $u(0)=u^{\prime}(0)=\ldots=u^{(k-1)}(0)=0$. It is a Hilbert space endowed with the norm: $\|u\|_{H_{\text {lef }}^{k}}=\left(\int_{0}^{1} u^{(k)}(x)^{2} \mathrm{~d} x\right)^{1 / 2}$. We denote by $\left(H_{\text {left }}^{k}\right)^{\prime}$ its topological dual space and by $\langle.,$. the corresponding duality bracket. Note that, so that no confusion arises, we also denote by $\langle.,$.$\rangle the distribution bracket.$

For any function of one of these spaces, we define the 'finite differentiation operator' $D_{n}$ by setting

$$
D_{n} u(x):= \begin{cases}n\left(u(x)-u\left(x-\frac{1}{n}\right)\right) & \text { if } x>\frac{1}{n} \\ n u(x) & \text { if } x \leq \frac{1}{n}\end{cases}
$$

and the operators of higher-order $D_{n}^{p}$ by setting $D_{n}^{0} u:=u$ and, for every positive integer $p$, $D_{n}^{p} u:=D_{n} D_{n}^{p-1} u$.

Using these notations, the deformation energies $E$ or $F$ (given by Equations (4) or (12)) of a pantograph or of a Warren-type pantographic structure can be written as

$$
\begin{aligned}
& E=K^{+} \frac{1}{n} \sum_{i=1}^{n}\left(D_{n}^{2}(v-w)\left(\frac{i}{n}\right)\right)^{2}+K^{-} \frac{1}{n} \sum_{i=1}^{n}\left(D_{n}^{2}(v+w)\left(\frac{i}{n}\right)\right)^{2} \\
& F=2 K \frac{1}{n} \sum_{i=1}^{n}\left(D_{n}^{3}(w)\left(\frac{i}{n}\right)\right)^{2} .
\end{aligned}
$$

Then the convergence of the total energy of a pantograph or of a Warren-type pantographic structure can easily be deduced from the convergence in $\mathcal{M}$ of the general functional:

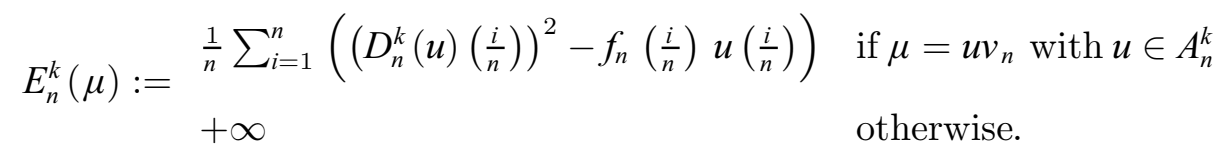


Here $f_{n}$ is defined $v_{n}$ almost everywhere and corresponds to a component of external forces. To deal with these external forces, we need to introduce the operators $I_{n}^{k}$ by setting for every function $f$ defined $v_{n}$ a.e.:

$$
I_{n} f\left(\frac{i}{n}\right):=\frac{1}{n} \sum_{j=i}^{n} f\left(\frac{j}{n}\right)
$$

$I_{n}^{0} f:=f$, and $I_{n}^{k+1} f:=I_{n} I_{n}^{k} f$. Then, for every $u \in A_{n}^{k}$, we have

$$
\frac{1}{n} \sum_{i=1}^{n} f\left(\frac{i}{n}\right) u\left(\frac{i}{n}\right)=\frac{1}{n} \sum_{i=1}^{n} I_{n}^{k} f\left(\frac{i}{n}\right) D_{n}^{k} u\left(\frac{i}{n}\right) .
$$

Note that this equality holds also for $u \in C_{c}^{\infty}(0,1]$ and $n$ large enough.

Using the Riesz representation theorem, for every $f \in H_{\text {left }}^{k}{ }^{\prime}$, there exists a unique $v \in H_{\text {left }}^{k}$ such that, for any $u \in H_{\text {left }}^{k}$

$$
\langle f, u\rangle=\int_{0}^{1} v^{(k)}(x) u^{(k)}(x) \mathrm{d} x
$$

By setting $I^{k} f:=v^{(k)}$ we define an operator $I^{k}$ from $H_{\text {left }}^{k}{ }^{\prime}$ on to $L^{2}(0,1)$ (when $k=1$ we simply denote it by $I$ ). For any $u \in H_{\text {left }}^{k}$, we have

$$
\langle f, u\rangle=\int_{0}^{1} I^{k} f(x) u^{(k)}(x) \mathrm{d} x .
$$

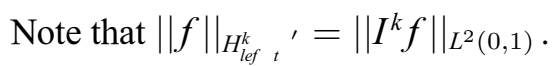

With these notations, our convergence result is:

Theorem 1 Assume that the sequence $\left(f_{n} v_{n}\right)$ converges weakly to somef in $\left(H_{l e f t}^{k}\right)^{\prime}$ and

$$
\lim _{n \rightarrow \infty} \frac{1}{n} \sum_{i=1}^{n}\left|I_{n}^{k} f_{n}\left(\frac{i}{n}\right)\right|^{2}=\int_{0}^{1}\left|I^{k} f(x)\right|^{2} \mathrm{~d} x .
$$

Then the sequence of functionals $\left(E_{n}^{k}\right) \Gamma$-converges to the functional $E^{k}$ defined by

$$
\begin{aligned}
& E^{k}(\mu):=\int_{0}^{1}\left(u^{(k)}(x)\right)^{2} \mathrm{~d} x-\langle f, u\rangle \quad \text { if } \mu=u \mathrm{~d} x \text { with } u \in H_{\text {left }}^{k} \\
& +\infty \quad \text { otherwise. }
\end{aligned}
$$

More precisely, for the weak* topology of $\mathcal{M}$, the following three statements hold.

(i) If $\left(\mu_{n}\right)$ is a sequence in $\mathcal{M}$ such that $\left(E_{n}^{k}\left(\mu_{n}\right)\right)$ is bounded, then $\left(\mu_{n}\right)$ is relatively compact.

(ii) Moreover, for any sequence $\left(\mu_{n}\right)$ converging to $\mu$, the following lower-bound inequality holds:

$$
\liminf _{n \longrightarrow \infty} E_{n}^{k}\left(\mu_{n}\right) \geq E^{k}(\mu) .
$$


(iii) Conversely, for every $\mu$ in $\mathcal{M}$, there exists a sequence $\left(\mu_{n}\right)$ converging to $\mu$ and satisfying the upper-bound inequality:

$$
\limsup _{n \longrightarrow \infty} E_{n}^{k}\left(\mu_{n}\right) \leq E^{k}(\mu) .
$$

The $\Gamma$-convergence is the mathematical notion which corresponds to the intuitive notion of convergence of state equations. It implies in particular the convergence of equilibrium solutions. Indeed (cf [46]) the following holds:

Corollary 1 Let $\mu_{n}$ be the minimizer of $E_{n}^{k}$ then any weak* limit of the sequence $\left(\mu_{n}\right)$ minimizes $E^{k}$.

\subsection{Proof of theorem 1}

We start this section with two auxiliary lemmas.

Lemma 1 (i) For every integer $k \geq 0$ and every $u \in A_{n}^{k}$, the following inequality holds

$$
\sup _{j=1 \ldots n}\left|u\left(\frac{j}{n}\right)\right|^{2} \leq \frac{1}{n} \sum_{i=1}^{n}\left|D_{n}^{k} u\left(\frac{i}{n}\right)\right|^{2} .
$$

(ii) For every $\varphi \in C_{c}^{\infty}(0,1]$, the following inequality holds

$$
\frac{1}{n} \sum_{i=1}^{n}\left|D_{n}^{k} \varphi\left(\frac{i}{n}\right)\right|^{2} \leq \int_{0}^{1}\left|\varphi^{(k)}(x)\right|^{2} \mathrm{~d} x .
$$

(iii) If $\left(u_{n} v_{n}\right)$ converges to $\mu$ in $\mathcal{M}$ then $D_{n}^{k} u_{n} v_{n}$ converges to the $k$ th derivative of $\mu$ in the sense of distributions on $(0,1)$.

Proof: Let $k^{\prime} \geq 0$. As $D_{n}^{k^{\prime}} u\left(\frac{1}{n}\right)=0$, for $j=1, \ldots, n$, we have

$$
D_{n}^{k^{\prime}} u\left(\frac{j}{n}\right)=\frac{1}{n} \sum_{i=1}^{j} D_{n}^{k^{\prime}+1} u\left(\frac{i}{n}\right) .
$$

Hence

$$
\left|D_{n}^{k^{\prime}} u\left(\frac{j}{n}\right)\right|^{2} \leq \frac{1}{n} \sum_{i=1}^{n}\left|D_{n}^{k^{\prime}+1} u\left(\frac{i}{n}\right)\right|^{2}
$$

and taking the sum over $j$

$$
\frac{1}{n} \sum_{i=1}^{n}\left|D_{n}^{k^{\prime}} u\left(\frac{i}{n}\right)\right|^{2} \leq \frac{1}{n} \sum_{i=1}^{n}\left|D_{n}^{k^{\prime}+1} u\left(\frac{i}{n}\right)\right|^{2} .
$$

This last inequality, together with Equation (26) in the case $k^{\prime}=0$, leads to assertion (i).

Now let $\psi \in C_{c}^{\infty}(0,1]$, by the Jensen inequality, we have 


$$
\int_{0}^{1}\left|D_{n} \psi(x)\right|^{2} \mathrm{~d} x \leq \int_{0}^{1} n \int_{x-\frac{1}{n}}^{x}\left|\psi^{\prime}(t)\right|^{2} \mathrm{~d} t \mathrm{~d} x=\int_{0}^{1} n\left[\int_{0}^{1} 1_{\left[t, t+\frac{1}{n}\right]}(x) \mathrm{d} x\right]\left|\psi^{\prime}(t)\right|^{2} \mathrm{~d} t
$$

which gives

$$
\int_{0}^{1}\left|D_{n} \psi(x)\right|^{2} \mathrm{~d} x \leq \int_{0}^{1}\left|\psi^{\prime}(x)\right|^{2} \mathrm{~d} x
$$

As the operator $D_{n}$ and the differentiation operator commute, a simple induction argument leads, for any positive integer $k^{\prime}$, to

$$
\int_{0}^{1}\left|D_{n}^{k^{\prime}} \psi(x)\right|^{2} \mathrm{~d} x \leq \int_{0}^{1}\left|\psi^{\left(k^{\prime}\right)}(x)\right|^{2} \mathrm{~d} x .
$$

Again, the Jensen inequality implies

$$
\frac{1}{n} \sum_{i=1}^{n}\left|D_{n} \psi\left(\frac{i}{n}\right)\right|^{2}=\frac{1}{n} \sum_{i=1}^{n}\left|n \int_{\frac{i-1}{n}}^{\frac{i}{n}} \psi^{\prime}(x) \mathrm{d} x\right|^{2} \leq \int_{0}^{1}\left|\psi^{\prime}(x)\right|^{2} \mathrm{~d} x .
$$

To prove Equation (25), we use the last inequality with $\psi=D_{n}^{k-1} \varphi$ :

$$
\frac{1}{n} \sum_{i=1}^{n}\left|D_{n}^{k} \varphi\left(\frac{i}{n}\right)\right|^{2} \leq\left.\int_{0}^{1} D_{n}^{k-1} \varphi^{\prime}(x)\right|^{2} \mathrm{~d} x
$$

Now applying inequality (27) with $\psi=\varphi^{\prime}$ and $k^{\prime}=k-1$, we obtain

$$
\left.\int_{0}^{1} D_{n}^{k-1} \varphi^{\prime}(x)\right|^{2} \mathrm{~d} x \leq\left.\int_{0}^{1} \varphi^{(k)}(x)\right|^{2} \mathrm{~d} x
$$

which completes the proof of assertion (ii).

Let us consider a sequence $\left(u_{n} v_{n}\right)$ converging to $\mu$ in $\mathcal{M}$. Letting $\varphi \in C_{c}^{\infty}(0,1)$, we have

$$
\begin{aligned}
& \lim _{n \rightarrow \infty} \frac{1}{n} \sum_{i=1}^{n} D_{n}^{k} u_{n}\left(\frac{i}{n}\right) \varphi\left(\frac{i}{n}\right)-\left\langle\mu^{(k)}, \varphi\right\rangle \\
= & \lim _{n \rightarrow \infty}(-1)^{k} \frac{1}{n} \sum_{i=1}^{n} u_{n}\left(\frac{i}{n}\right) D_{n}^{k} \varphi\left(\frac{i-k}{n}\right)-(-1)^{k}\left\langle\mu, \varphi^{(k)}\right\rangle \\
= & (-1)^{k} \lim _{n \rightarrow \infty}\left[\frac{1}{n} \sum_{i=1}^{n} u_{n}\left(\frac{i}{n}\right)\left(D_{n}^{k} \varphi\left(\frac{i-k}{n}\right)-\varphi^{(k)}\left(\frac{i}{n}\right)\right)\right] .
\end{aligned}
$$


Since $\sup _{i}\left|D_{n}^{k} \varphi\left(\frac{i-k}{n}\right)-\varphi^{(k)}\left(\frac{i}{n}\right)\right|$ tends to zero as $n$ tends to infinity, the last limit converges to zero. Assertion (iii) is proved.

The following lemma collects some preliminary results for operators $I_{n}^{k}$.

Lemma 2 For any $\Phi \in C_{c}^{\infty}[0,1]$, we have

$$
\lim _{n \rightarrow \infty} \frac{1}{n} \sum_{i=1}^{n}\left|I_{n}^{k} \Phi\left(\frac{i}{n}\right)-I^{k} \Phi\left(\frac{i}{n}\right)\right|^{2}=0 .
$$

Let $\left(f_{n} v_{n}\right)$ be a sequence converging to $f$ weakly in $H_{\text {left }}^{k}{ }^{\prime}$ and satisfying

$$
\lim _{n \rightarrow \infty} \frac{1}{n} \sum_{i=1}^{n}\left|I_{n}^{k} f_{n}\left(\frac{i}{n}\right)\right|^{2}=\int_{0}^{1}\left|I^{k} f(x)\right|^{2} \mathrm{~d} x .
$$

Then, for any $\varphi \in C_{c}^{\infty}(0,1]$, we have

$$
\lim _{n \rightarrow \infty} \frac{1}{n} \sum_{i=1}^{n} I_{n}^{k} f_{n}\left(\frac{i}{n}\right) \varphi\left(\frac{i}{n}\right)=\int_{0}^{1} I^{k} f(x) \varphi(x) \mathrm{d} x .
$$

Proof: For a regular function $\Phi, I \Phi(x)$ coincides with the integral $\int_{x}^{1} \Phi(t) \mathrm{d} t$. Then, for some constant $C$, we have

$$
\sup _{i}\left|I_{n} \Phi\left(\frac{i}{n}\right)-I \Phi\left(\frac{i}{n}\right)\right| \leq \frac{C}{n},
$$

which proves Equation (28) in the particular case of $k=1$. On the other hand, for all $\Phi$ defined $v_{n}$ a.e.,

$$
\sup _{i}\left|I_{n} \Phi\left(\frac{i}{n}\right)\right| \leq \sup _{i}\left|\Phi\left(\frac{i}{n}\right)\right| .
$$

Applying inequality (32) to $\widetilde{\Phi}=I_{n}^{k} \Phi-I^{k} \Phi$ and inequality (31) to $\widetilde{\Phi}=I^{k}(\Phi)$, we obtain

$$
\begin{aligned}
& \sup _{i}\left|I_{n}^{k+1} \Phi\left(\frac{i}{n}\right)-I^{k+1} \Phi\left(\frac{i}{n}\right)\right| \\
\leq & \sup _{i}\left|I_{n}\left(I_{n}^{k} \Phi-I^{k} \Phi\right)\left(\frac{i}{n}\right)\right|+\sup _{i} \mid I_{n}\left(I^{k} \Phi\left(\frac{i}{n}\right)-I\left(I^{k} \Phi\right)\left(\frac{i}{n}\right) \mid\right. \\
\leq & \sup _{i}\left|I_{n}^{k} \Phi\left(\frac{i}{n}\right)-I^{k} \Phi\left(\frac{i}{n}\right)\right|+\frac{C}{n} .
\end{aligned}
$$

Proof of Equation (28) is then obtained by an induction argument.

Letting $\psi \in C_{c}^{\infty}(0,1]$ such that $\varphi=\psi^{(k)}$, we have 


$$
\begin{aligned}
& \frac{1}{n} \sum_{i=1}^{n} I_{n}^{k} f_{n}\left(\frac{i}{n}\right) \psi^{(k)}\left(\frac{i}{n}\right)-\int_{0}^{1} I^{k} f(x) \psi^{(k)}(x) \mathrm{d} x \\
= & \frac{1}{n} \sum_{i=1}^{n} I_{n}^{k} f_{n}\left(\frac{i}{n}\right) D_{n}^{k} \psi\left(\frac{i}{n}\right)-\int_{0}^{1} I^{k} f(x) \psi^{(k)}(x) \mathrm{d} x \\
- & \frac{1}{n} \sum_{i=1}^{n} I_{n}^{k} f_{n}\left(\frac{i}{n}\right)\left(D_{n}^{k} \psi\left(\frac{i}{n}\right)-\psi^{(k)}\left(\frac{i}{n}\right)\right) \\
= & \left\langle f_{n} v_{n}-f, \psi\right\rangle-\frac{1}{n} \sum_{i=1}^{n} I_{n}^{k} f_{n}\left(\frac{i}{n}\right)\left(D_{n}^{k} \psi\left(\frac{i}{n}\right)-\psi^{(k)}\left(\frac{i}{n}\right)\right) .
\end{aligned}
$$

These last two terms tend to zero since $\left(f_{n} v_{n}\right)$ converges weakly to $f$, the quantity $\frac{1}{n} \sum_{i=1}^{n}\left|I_{n}^{k} f_{n}\left(\frac{i}{n}\right)\right|^{2}$ is bounded and the function $D_{n}^{k} \varphi-\varphi^{(k)}$ converges uniformly to zero. Hence, Equation (30) is proved.

\section{Proof of theorem 1:}

(i) Relative compactness. Let $\left(u_{n} v_{n}\right)$ be a sequence of bounded energy. Using the CauchySchwarz inequality and Equation (18), we have

$$
\begin{aligned}
\frac{1}{n} \sum_{i=1}^{n} f_{n}\left(\frac{i}{n}\right) u_{n}\left(\frac{i}{n}\right) & =\frac{1}{n} \sum_{i=1}^{n} I_{n}^{k} f_{n}\left(\frac{i}{n}\right) D_{n}^{k} u_{n}\left(\frac{i}{n}\right) \\
& \leq\left(\frac{1}{n} \sum_{i=1}^{n}\left|I_{n}^{k} f_{n}\left(\frac{i}{n}\right)\right|^{2}\right)^{\frac{1}{2}}\left(\frac{1}{n} \sum_{i=1}^{n}\left|D_{n}^{k} u_{n}\left(\frac{i}{n}\right)\right|^{2}\right)^{\frac{1}{2}}
\end{aligned}
$$

Then the boundedness of $E_{n}^{k}\left(u_{n} v_{n}\right)$ and $\frac{1}{n} \sum_{i=1}^{n}\left|I_{n}^{k} f_{n}\left(\frac{i}{n}\right)\right|^{2}$ imply that

$$
\frac{1}{n} \sum_{i=1}^{n}\left|D_{n}^{k} u_{n}\left(\frac{i}{n}\right)\right|^{2} \leq M^{2}
$$

for some constant $M$. Lemma 1(i) implies that $u_{n}$ is uniformly bounded by $M$. So is the total variation of the measure $u_{n} v_{n}$. Then the sequence $\left(u_{n} v_{n}\right)$ is relatively compact with respect to the weak* topology of $\mathcal{M}$.

(ii) Lower bound. Let $\left(u_{n} v_{n}\right)$ be a sequence of bounded energy converging to some $\mu$ in $\mathcal{M}$ and consider $\Phi \in C_{c}^{\infty}[0,1]$. Using Lemma 2, we can write

$$
\begin{aligned}
\left|\left\langle\mu, \Phi^{(k)}\right\rangle\right|^{2} & =\lim _{n \rightarrow \infty}\left|\frac{1}{n} \sum_{i=1}^{n} u_{n}\left(\frac{i}{n}\right) \Phi^{(k)}\left(\frac{i}{n}\right)\right|^{2} \\
& =\lim _{n \rightarrow \infty}\left|\frac{1}{n} \sum_{i=1}^{n} D_{n}^{k} u_{n}\left(\frac{i}{n}\right) I_{n}^{k} \Phi^{(k)}\left(\frac{i}{n}\right)\right|^{2}
\end{aligned}
$$




$$
\begin{aligned}
& \leq \liminf _{n \rightarrow \infty}\left(\frac{1}{n} \sum_{i=1}^{n}\left|D_{n}^{k} u_{n}\left(\frac{i}{n}\right)\right|^{2}\right)\left(\frac{1}{n} \sum_{i=1}^{n}\left|I_{n}^{k} \Phi^{(k)}\left(\frac{i}{n}\right)\right|^{2}\right) \\
& \leq \liminf _{n \rightarrow \infty}\left(\frac{1}{n} \sum_{i=1}^{n}\left|D_{n}^{k} u_{n}\left(\frac{i}{n}\right)\right|^{2}\right) \int_{0}^{1}|\Phi(x)|^{2} \mathrm{~d} x .
\end{aligned}
$$

This last inequality implies the existence of a unique $u \in H_{\text {left }}^{k}$ such that $\mu=u \mathrm{~d} x$. Moreover, it implies that

$$
\liminf _{n \rightarrow \infty}\left(\frac{1}{n} \sum_{i=1}^{n}\left|D_{n}^{k} u_{n}\left(\frac{i}{n}\right)\right|^{2}\right) \geq \int_{0}^{1}\left|u^{(k)}(x)\right|^{2} \mathrm{~d} x .
$$

For any $\varepsilon>0$, let $F_{\varepsilon} \in C_{c}^{\infty}(0,1)$ be such that $\int_{0}^{1}\left|I^{k} f(x)-F_{\varepsilon}(x)\right|^{2} \mathrm{~d} x<\varepsilon^{2}$. We have

$$
\left|\langle f, u\rangle-\int_{0}^{1} F_{\varepsilon}(x) u^{(k)}(x) \mathrm{d} x\right|=\left|\int_{0}^{1}\left(I^{k} f-F_{\varepsilon}\right)(x) u^{(k)}(x) \mathrm{d} x\right| \leq \varepsilon M .
$$

Moreover, by Lemma 1(iii), we have

$$
\lim _{n \rightarrow \infty}\left|\int_{0}^{1} F_{\varepsilon}(x) u^{(k)}(x) \mathrm{d} x-\frac{1}{n} \sum_{i=1}^{n} F_{\varepsilon}\left(\frac{i}{n}\right) D_{n}^{k} u_{n}\left(\frac{i}{n}\right)\right|=0,
$$

and by Lemma 2

$$
\begin{aligned}
& \limsup _{n \rightarrow \infty}\left(\frac{1}{n} \sum_{i=1}^{n}\left(I_{n}^{k} f_{n}\left(\frac{i}{n}\right)-F_{\varepsilon}\left(\frac{i}{n}\right)\right) D_{n}^{k} u_{n}\left(\frac{i}{n}\right)\right)^{2} \\
\leq & M^{2} \limsup _{n \rightarrow \infty} \frac{1}{n} \sum_{i=1}^{n}\left|I_{n}^{k} f_{n}\left(\frac{i}{n}\right)-F_{\varepsilon}\left(\frac{i}{n}\right)\right|^{2} \\
\leq & M^{2} \limsup _{n \rightarrow \infty} \frac{1}{n} \sum_{i=1}^{n}\left|I_{n}^{k} f_{n}\left(\frac{i}{n}\right)\right|^{2}-2 I_{n}^{k} f_{n}\left(\frac{i}{n}\right) F_{\varepsilon}\left(\frac{i}{n}\right)+\left|F_{\varepsilon}\left(\frac{i}{n}\right)\right|^{2} \\
\leq & M^{2} \int_{0}^{1}\left(\left|I^{k} f(x)\right|^{2}-2 I^{k} f(x) F_{\varepsilon}(x)+\left|F_{\varepsilon}(x)\right|^{2}\right) \mathrm{d} x \\
\leq & M^{2} \int_{0}^{1}\left|I^{k} f(x)-F_{\varepsilon}(x)\right|^{2} \mathrm{~d} x \leq M^{2} \varepsilon^{2} .
\end{aligned}
$$

As $\varepsilon$ is arbitrary, collecting Equations (35), (36) and (37) gives 


$$
\lim _{n \rightarrow \infty} \frac{1}{n} \sum_{i=1}^{n} f_{n}\left(\frac{i}{n}\right) u_{n}\left(\frac{i}{n}\right)=\lim _{n \rightarrow \infty} \frac{1}{n} \sum_{i=1}^{n} I_{n}^{k} f_{n}\left(\frac{i}{n}\right) D_{n}^{k} u_{n}\left(\frac{i}{n}\right)=\langle f, u\rangle
$$

which, together with Equation (34), proves the lower-bound inequality.

(iii) Upper bound. Now consider $\mu \in \mathcal{M}$ such that $E^{k}(\mu)<\infty$. Then there exists $u \in H_{\text {left }}^{k}$ such that $\mu=u \mathrm{~d} x$. Using a density argument we can assume that $u$ belongs to $C_{c}^{\infty}(0,1]$. For $n$ large enough, let us define $u_{n} \in A_{n}^{k}$ by setting, for all $i \in\{1, \ldots, n\}, u_{n}\left(\frac{i}{n}\right):=u\left(\frac{i}{n}\right)$. Clearly $u_{n} v_{n}$ converges in $\mathcal{M}$ to $u \mathrm{~d} x$. Since $f_{n} v_{n}$ converges to $f$ in $H_{\text {left }}^{k}{ }^{\prime}$, we have

$$
\lim _{n \rightarrow \infty} \frac{1}{n} \sum_{i=1}^{n} f_{n}\left(\frac{i}{n}\right) u_{n}\left(\frac{i}{n}\right)=\langle f, u\rangle
$$

Moreover, using Lemma 1(ii), we have

$$
\limsup _{n \rightarrow \infty} \frac{1}{n} \sum_{i=1}^{n}\left|D_{n}^{k} u_{n}\left(\frac{i}{n}\right)\right|^{2} \leq \int_{0}^{1}\left|u^{(k)}(x)\right|^{2} \mathrm{~d} x .
$$

The upper-bound inequality results from Equations (39) and (40).

\section{MECHANICS OF HIGHER GRADIENT BEAMS}

For the considered pantographic structures, we have established mathematically the validity of the continuous models introduced by functionals (13) and (5). In this section we briefly discuss the mechanical properties of such limit models paralleling the treatment developed in [47]. Some new boundary conditions have to be given for determining uniquely the solution of the corresponding Euler-Lagrange equations. We interpret these in terms of the underlying discrete models, double (or triple) forces, which can be hardly understood in the context of homogenized models. We find easily a microscopic counterpart and their physical meaning is clarified.

\subsection{Second gradient beams}

Let us call pantographic beam the homogenized model for the pantographic structure. Its reference unstressed configuration is a straight segment of length one. Owing to Theorem 1 we can write its deformation energy in the following form

$$
\mathcal{E}(v, w)=\frac{\alpha}{2} \int_{0}^{1}\left(\left(v^{\prime \prime}\right)^{2}-2 \beta v^{\prime \prime} w^{\prime \prime}+\left(w^{\prime \prime}\right)^{2}\right) \mathrm{d} x
$$

where $v, w$ correspond respectively to the axial displacement and deflection, $\alpha:=2\left(K^{+}+\right.$ $\left.K^{-}\right)$is a positive parameter and $\beta:=\left(K^{-}-K^{+}\right)\left(K^{+}+K^{-}\right)^{-1}$ satisfies $|\beta|<1$. Moreover, the beam is clamped at $x=0$; the displacement $(v, w)$ and its first derivative $\left(v^{\prime}, w^{\prime}\right)$ vanish at that point. 
Clearly, as the energy depends on the second gradient of the displacement, this is a 1D model of a second gradient material body. The fact that no classical elastic term (involving the first derivatives of the displacement) appears in the energy is due to our choice of a very particular structure - the pantograph. It could have been easy to get such terms by adding extensible bars linking nodes $C_{i}, C_{i+1}$. The term $\left(w^{\prime \prime}\right)^{2}$ describes the standard bending behaviour of the beam while the terms involving $v$ " are less usual; these were first studied by Casal [19] (see also [20]). Our example of the homogenized pantographic beam gives a possible microscopic interpretation for Casal's second gradient extensible beam.

We start considering the case $\beta=0$ where axial deformation and deflection are uncoupled and we focus on purely axial displacements $(w=0)$. The beam is statically determined due to the boundary conditions

$$
v(0)=0, \quad v^{\prime}(0)=0 .
$$

Example 1. Let us assume that the beam is subjected to a continuous linear density of external axial forces $f$ and a concentrated axial force $F$ at endpoint $x=1$. At equilibrium, the displacement field minimizes

$$
\int_{0}^{1}\left(\frac{\alpha}{2}\left(v^{\prime \prime}\right)^{2}-f v\right) \mathrm{d} x-F v(1)
$$

under the constraints (42). Then it satisfies

$$
\alpha v^{\prime \prime \prime \prime}=f \text { on }(0,1), \quad v(0)=v^{\prime}(0)=0, \quad v^{\prime \prime}(1)=0, \quad-\alpha v^{\prime \prime \prime}(1)=F .
$$

If we assume that $F=0$ and that $f$ is vanishing in, say, $(1 / 2,1)$ and $f \geq 0$ elsewhere, then the solution $v$ satisfies $v \geq 0$ and $v^{\prime}$ is a positive constant in $(1 / 2,1)$. Let us interpret this situation by considering the original pantograph; its nodes $i<n / 2$ are submitted to external forces $f(i / n)$, the pantograph is extended and has the peculiar property that this extension propagates in the free part $i \geq n / 2$ of the pantograph. If $f=0$ and $F$ is non-vanishing then the constitutive relation for contact forces and the extensible displacement field are quite different from the classical extensible bar. Indeed we find that axial contact forces fix the third derivative of the displacement field; moreover, the displacement is a cubic function while it is linear in the classical case.

Example 2. Let us assume now that the pantograph is only subject to two opposite axial forces $n G$ and $-n G$ applied respectively at nodes $C_{n}$ and $C_{n-1}$. The resulting force and the moment of this 'pair of forces' (or, to avoid confusion, of this double force [10] or double traction [19]) vanish. However, this double force has a great influence upon the equilibrium state. It expends work on any variation of the distance $C_{n-1} C_{n}$. The extension of the last pantographic module tends to propagate in the whole pantograph and is only limited by the clamping constraints at the other endpoint. The correspondence with the homogenized point of view is clear. The applied forces converge in the sense of Theorem 1 to the distribution of order one $-G \delta_{1}^{\prime}$ and the equilibrium displacement field of the homogenized beam minimizes 


$$
\int_{0}^{1} \alpha\left(v^{\prime \prime}\right)^{2} \mathrm{~d} x-G v^{\prime}(1)
$$

under the constraints (42). Then it satisfies

$$
\alpha v^{\prime \prime \prime \prime}=0 \text { on }(0,1), \quad v(0)=v^{\prime}(0)=0, \quad v^{\prime \prime \prime}(1)=0, \quad \alpha v^{\prime \prime}(1)=G
$$

the solution of which is $v(x)=G x^{2} / 2 \alpha$. This example enlightens the physical meaning of the unusual boundary condition $\alpha \nu$ " $(1)=G$ which naturally appears in any second gradient theory [10]. It becomes clear why, in such a boundary condition, $G$ is called a 'double force'.

In the case $\beta \neq 0$ the axial deformation and the deflection are coupled but the equilibrium state is still easy to compute. Let us reconsider Example 2 in this case.

Example 3. $\beta \neq 0$ and the pantograph is still only subject to two opposite traction forces $n G$ and $-n G$ applied respectively at nodes $C_{n}$ and $C_{n-1}$. The equilibrium displacement field $(v, w)$ of the homogenized beam minimizes

$$
\frac{\alpha}{2} \int_{0}^{1}\left(\left(v^{\prime \prime}\right)^{2}-2 \beta v^{\prime \prime} w^{\prime \prime}+\left(w^{\prime \prime}\right)^{2}\right) \mathrm{d} x-G v^{\prime}(1)
$$

under the constraints $v(0)=v^{\prime}(0)=w(0)=w^{\prime}(0)=0$. Then we have

$$
\frac{w(x)}{\beta}=v(x)=\frac{G x^{2}}{2\left(1-\beta^{2}\right)} \quad \text { on }(0,1) .
$$

The double force applied at endpoint $x=1$ is able to bend and extend the beam even if the applied resultant force and moment vanish.

\subsection{Third gradient beams}

Let us now consider a Warren-type pantographic structure of length one. The associated homogenized beam is inextensible $(v=0)$, its reference unstressed configuration is a straight segment of length one and, as a consequence of Theorem 1, its deformation energy has the following form

$$
\mathcal{E}(v, w)=\frac{\alpha}{2} \int_{0}^{1}\left(w^{\prime \prime \prime}\right)^{2} \mathrm{~d} x
$$

where $w$ corresponds the deflection, and $\alpha:=4 K$ is a positive parameter. Moreover, as we have assumed that the beam is clamped at $x=0$, the displacement and its first two derivatives vanish at this point $\left(w(0)=w^{\prime}(0)=w^{\prime \prime}(0)=0\right)$. This is a 1D model of a third gradient material body.

Example 4. Let us assume that the pantographic Warren-type structure is subject either to a single transverse force $F$ at node $A_{n}$ or to two transverse forces $n G,-n G$ at nodes $A_{n}$, $A_{n-1}$ or to three transverse forces $n^{2} H,-2 n^{2} H, n^{2} H$ at nodes $A_{n}, A_{n-1}, A_{n-2}$, or eventually 
to the sum of these different actions. $F$ is a shear force which tends to displace the endpoint $y$ of the structure while $G$ is a couple which tends to rotate this extremity. $H$ could be called either triple force or double couple; it tends to curve the extremity of the structure. From the point of view of the homogenized beam, one has to minimize

$$
\frac{\alpha}{2} \int_{0}^{1}\left(w^{\prime \prime \prime}\right)^{2} \mathrm{~d} x-F w(1)-G w^{\prime}(1)-H w^{\prime \prime}(1)
$$

under the constraint $w(0)=w^{\prime}(0)=w^{\prime \prime}(0)=0$. The corresponding Euler-Lagrange equation is a sixth-order differential equation which, together with the boundary conditions, reads

$$
\begin{aligned}
-\alpha w^{(6)} & =0 \text { on }(0,1), \\
w(0) & =w^{\prime}(0)=w^{\prime \prime}(0)=0, \\
\alpha v^{\prime \prime \prime}(1) & =H,-\alpha v^{(4)}(1)=G, \alpha v^{(5)}(1)=F .
\end{aligned}
$$

$H$ is a special feature of third gradient models. It represents a new kind of contact action. Note that at the other endpoint the kinematic dual condition $w^{\prime \prime}(0)=0$ fixes locally the curvature of the beam.

Example 5. Let us assume that only the first nodes $i<n / 2$ of the pantographic Warrentype structure are subject to transverse external forces $f(i / n)$, where $f$ is a given continuous function vanishing on $(1 / 2,1)$. From the point of view of the homogenized beam, one has to solve the following differential equation:

$$
\begin{aligned}
-\alpha w^{(6)} & =f \text { on }(0,1) \\
w(0) & =w^{\prime}(0)=w^{\prime \prime}(0)=0, \\
v^{(3)}(1) & =v^{(4)}(1)=v^{(5)}(1)=0 .
\end{aligned}
$$

We note that the free part $x \geq 1 / 2$ of the beam has a constant, generally non-vanishing, curvature.

Acknowledgments. This research was partly supported by visiting grants from both the Universite de Toulon et du Var and the Università di Roma La Sapienza.

\section{REFERENCES}

[1] Noll, W.: The Foundations of Mechanics and Thermodynamics, selected papers, 1974.

[2] Eringen, A. C.: A unified theory of thermomechanical materials, Int. J. Eng. Sci., 4, 179-202 (1966).

[3] Gurtin, M.: Thermodynamics and the possibility of spatial interaction in elastic materials. Arch. Ration. Mech. Anal., 19, 339-352 (1965).

[4] Dunn, J. E. and Serrin, J.: On the thermomechanics of interstitial working. Arch. Ration. Mech. Anal., 88, 95-133 (1985).

[5] Mindlin, R. D. and Tiersten, H. F.: Effects of couple-stresses in linear elasticity. Arch. Ration. Mech. Anal., 11, 415-448 (1962). 
72 J.-J. ALIBERT ET AL.

[6] Capriz, G.: Continua with constrained or latent microstructure. Material instabilities in continuum mechanics (Edinburgh, 1985-1986), 53-64, Oxford Science Publications, Oxford University Press, New York, 1988.

[7] Batra, R. C.: Thermodynamics of non-simple elastic materials. J. Elasticity, 6, 451-456 (1976).

[8] Maugin, G. A.: Material inhomogeneities in elasticity. Applied Mathematics and Mathematical Computation, 3, Chapman and Hall, London, 1993.

[9] Daher, N. and Maugin, G. A.: The method of virtual power in continuum mechanics. Application to media presenting singular surfaces and interfaces. Acta Mech. 60, 217-240 (1986).

[10] Germain, P.: La méthode des puissances virtuelles en mécanique des milieux continus. I. Théorie du second gradient. J. Mécanique, 12, 235-274 (1973).

[11] Toupin, R. A.: Elastic materials with couple stress. Arch. Ration. Mech. Anal., 11, 385-414 (1962).

[12] Germain, P.: The method of virtual power in continuum mechanics: II. Microstructure. Collection of articles dedicated to William Prager on the occasion of his seventieth birthday. (A. C. Pipkin ed) SIAM J. Appl. Math. 25, 556-575 (1973).

[13] Vlasov, V. Z.: Thin-Walled Elastic Beams, NSF Publication, 1961.

[14] Timoshenko, S.: Strength of Materials, Parts I and II, Krieger Publishing, 1976.

[15] Kachanov, L. M.: Buckling of Thin-Walled Rods Leipholz University of Waterloo Press, Waterloo, Canada, 1983.

[16] Cosserat, E. and Cosserat, F.: Théorie des Corps Déformables, Hermann, Paris, 1909.

[17] Ehlers, W.: Constitutive equations for granular materials in geomechanical context. Continuum mechanics in environomental sciences and geophysics. CISM Courses and Lectures, 337, 313-402, Springer, Vienna, 1993.

[18] Cahn, J. W. and Hilliard, J. E.: Free energy of a non-uniform system, I. Interfacial free energy. J. Chem. Phys., 28, 258-267 (1958).

[19] Casal, P.: La capillarité interne. Cahier du Groupe Français d'Etudes de Rheologie. CNRS VI, 31-37 (1961).

[20] Vardoulakis, I., Exadaktylos, G. and Aifantis, E.: Gradient elasticity with surface energy: Mode-III crack problem. Int. J. Solids Struct., 33, 4531-4559 (1996).

[21] Muhlhaus, H. B. and Aifantis, E.: A variational principle for gradient plasticity. Int. J. Solids Struct., 28, 845-857 (1991).

[22] Frémond, M. and Nedjar, B.: Damage, Gradient of Damage and principle of virtual power. Int. J. Solids Struct., 33, 1083-1103 (1996).

[23] Batra, R. C. and Hwang, J.: An adaptive mesh refinement technique for two-dimensional shear band problems. Comput. Mech., 12, 255-268 (1993).

[24] Batra, R. C. and Hwang, J.: Dynamic shear band development in dipolar thermoviscoplastic materials. Comput. Mech., 12, 354-369 (1994).

[25] Maugin, G. A.: Nonlinear Waves in Elastic Crystals, Oxford Science Publications, 1999.

[26] Zbirohowski-Koscia, K.: Thin-Walled Beams from Theory to Practice, Crosby Lockwood, London, 1967.

[27] Bensoussan, A., Lions, J. L. and Papanicolaou, G.: Asymptotics Analysis for Periodic Structures, North-Holland, Amsterdam, 1978.

[28] Cioranescu, D. and Saint Jean Paulin, J.: Homogenization of Reticulated Structures, Applied Mathematical Sciences 136, Springer-Verlag, New York, 1999.

[29] Banks, H. T., Cioranescu, D. and Rebnord, D. A.: Homogenization models for two-dimensional grid structures. Asymptotic Anal. 11, 107-130 (1995).

[30] Ciarlet, P. G., Trabucho, L. and Viaño, J. M.: Proc. Int. Conf. Asymptotic Methods for Elastic Structures (Lisbon, 4-8 October 1993) de Gruyter \& Co, Berlin, 1995.

[31] Figueiredo, I. N. and Trabucho, L.: A Galerkin approximation for linear elastic shallow shells. Comput. Mech. 10, 107-119 (1992).

[32] Trabucho, L. and Viaño, J. M.: Mathematical modelling of rods. Handbook of Numerical Analysis, vol IV, 487-974, North-Holland, Amsterdam, 1996.

[33] Lewiǹski, T. and Telega, J. J.: Plates, laminates and shells. Asymptotic Analysis and Homogenization. Series on Advances in Mathematics for Applied Sciences 52, World Scientific, River Edge, NJ, 2000.

[34] Benvenuto, E.: An Introduction to the History of Structural Mechanics, Springer-Verlag, Berlin, 1991.

[35] Clebsch, A.: Théorie de l'élasticité des corps solides (Traduite par. MM. Barré de Saint-Venant et Flamant, avec des Notes étendue de M. Barré de Saint-Venant), Dunod, Paris 1883 (reprinted by Johnson Reprint Corporation, New York, 1966).

[36] Noor, A. K. and Andersen, C. M.: Analysis of beam-like lattice trusses Comp. Meth. Appl. Mech. Eng., 2, 53-70 (1979). 
[37] Di Carlo, A., Rizzi, N. and Tatone. A.: Continuum modelling of beam-like latticed truss: identification of the constitutive functions for the contact and inertial actions. Meccanica, 25, 168-174 (1990).

[38] Dillon, O. W. and Perzyna, P.: A gradient theory of materials with memory and internal changes. Arch. Mech. Stosow., 24, 7270-747 (1972).

[39] Cioranescu, D. and Saint Jean Paulin, J.: Stuctures très minces en élasticité linéarisée: tours et grillages. C.R. Acad. Sci. Paris I, 308, 41-46 (1989).

[40] Cioranescu, D. and Saint Jean Paulin, J.: Mathematical study of large space structures, Lecture notes in Control and Inform. Sci., 147, Springer-Verlag, Berlin, 1990.

[41] Cioranescu, D. and Saint Jean Paulin, J.: Truss structures: Fourier conditions and eigenvalue problem, Lecture notes in Control and Inform. Sci., 178, Springer-Verlag, Berlin, 1992.

[42] De Giorgi, E.: Some remarks on $\Gamma$-convergence and least-squares method. Composite media and homogenization theory (Trieste, 1990). Progress in Nonlinear Differential Equations and their Applications, 5, 135-142, Birkhäuser Boston, Boston, 1991.

[43] Colombini, F., Marino, A., Modica, L. and Spagnolo, S. ed: Partial differential equations and the calculus of variations, vol II. Essays in honour of Ennio De Giorgi. Progress in Nonlinear Differential Equations and their Applications, 2, Birkhäuser Boston, Boston, 1989.

[44] Colombini, F., Marino, A., Modica, L. and Spagnolo, S. ed: Partial differential equations and the calculus of variations, vol I. Essays in honour of Ennio De Giorgi. Progress in Nonlinear Differential Equations and their Applications, 1, Birkhäuser Boston, Boston, 1989.

[45] Braides, A. and Gelli, M. S.: Limits of discrete systems with long-range interactions. Preprint SISSA, 41/99/M (1999).

[46] Dal Maso, G.: Introduction to $\Gamma$-convergence, Progress in Nonlinear Differential Equations and their Applications, 8, Birkhäuser, Boston, 1993.

[47] Washizu, K.: Variational Methods in Elasticity and Plasticity, Pergamon Press, Oxford, 1975.

[48] Wright, T. W. and Batra, R. C.: Adiabatic shear bands in simple and dipolar plastic materials, Macro- and MicroMechanics of High Velocity Deformation and Fracture, IUTAM Symposium on MMMHVDF (Tokyo, Japan, 12-15 August 1985) Springer-Verlag, Berlin, 1987.

[49] Batra, R. C. and Chen, L.: Instability analysis and shear band spacing in gradient-dependent thermoviscoplastic materials with finite speeds of thermal waves. Arch. Mech. 53, 167-192 (2001). 